\title{
Intrinsic correlation between fragility and bulk modulus in metallic glasses
}

\author{
Minqiang Jiang ${ }^{1}$ and Lanhong Dai ${ }^{1,2, *}$ \\ ${ }^{1}$ State Key Laboratory of Nonlinear Mechanics, Institute of Mechanics, Chinese Academy of Sciences, \\ Beijing 100080, People's Republic of China \\ ${ }^{2}$ State Key Laboratory of Explosion Science and Technology, Beijing 100081, People's Republic of China \\ (Received 23 January 2007; revised manuscript received 10 April 2007; published 14 August 2007)
}

\begin{abstract}
A systematic study on the available data of 26 metallic glasses shows that there is an intrinsic correlation between fragility of a liquid and bulk modulus of its glass. The underlying physics can be rationalized within the formalism of potential energy landscape thermodynamics. It is surprising to find that the linear correlation between the fragility and the bulk-shear modulus ratio exists strictly at either absolute zero temperature or very high frequency. Further analyses indicate that a real flow event in bulk metallic glasses is shear dominant, and fragility is in inverse proportion to shear-induced bulk dilatation. Finally, extension of these findings to nonmetallic glasses is discussed.
\end{abstract}

DOI: 10.1103/PhysRevB.76.054204

PACS number(s): 64.70.Pf, 62.20.Dc, 61.43.Dq

\section{INTRODUCTION}

Understanding of the material-dependent slowing down dynamics is one of the central subjects in glass physics. In terms of the concept of fragility introduced by Angell, ${ }^{1}$ the glass-forming systems can be classified into "strong" or "fragile" pattern. The kinetic fragility $m$ is defined as

$$
m=\left.\frac{\partial \log \left(\eta / \eta_{\infty}\right)}{\partial\left(T_{g} / T\right)}\right|_{T=T_{g}},
$$

where $\eta_{\infty}$ is the limiting high-temperature shear viscosity and $T_{g}$ is the glass transition temperature. Here, the dimensionless fragility index, a measure of deviation from the Arrhenius behavior in the temperature-dependent viscosity, opens a new window into the understanding of glass transition and its slowing down dynamics. The kinetic fragility was found to be correlated with other properties characterizing the liquid side of glass transition ${ }^{2}$ and low-temperature properties of glasses such as vibration. ${ }^{3,4}$ An unexpected linear correlation between bulk-shear modulus ratio $\left(K_{\infty} / G_{\infty}\right)$ and fragility in nonmetallic glass was reported by Novikov and Sokolov. ${ }^{5}$ Due to the technological and the scientific significance of this surprising finding, it has attracted a lot of discussions. ${ }^{6-10}$ However, the question whether such a linear correlation universally exists still remains open up to now. As for metallic glasses, a number of important correlations among their properties have been revealed. ${ }^{11-16}$ Recently, Novikov and Sokolov ${ }^{17}$ discovered that there is also a linear correlation between the bulk-shear modulus ratio and fragility in metallic glasses, and the deviation from the linear correlation is due to the material-specific free electron gas. In this paper, a systematic study on the correlation between fragility and elastic modulus in metallic glasses is done, and further, its underlying physics is discussed.

\section{EXPERIMENTAL OBSERVATIONS}

Table I lists the measured physical properties of 26 metallic glasses, ${ }^{18}$ such as density $\rho$, molar mass $M$, bulk modulus $K$, shear modulus $G$, glass transition temperature $T_{g}$, and fragility $m$. It is well known that values of $m$ can be directly taken by using the Vogel-Fulcher-Tamman (VFT) equation fits to viscosity or relaxation time data. ${ }^{1}$ This is the kinetic method. On the other hand, the liquid fragility can be determined from a purely thermodynamic way, i.e., the heating rate dependent $T_{g} .{ }^{15,20}$ Let us note that the fragility values of metallic glasses numbered as 1-8, 14, 15, 17, and 18 in Table I are given by the thermodynamic method, whereas the others are obtained from viscosity or relaxation data. Besides, we also note that the fragility values are mostly derived from the latest literature such as Refs. 9, 15, and 17. Previous studies have demonstrated that the heating rate dependent $T_{g}$ describes the fragility equally well as complementary viscosity or relaxation time measurements. ${ }^{29-31}$ Thus, the following results according to these values in Table I are believable, and the unavoidable slight error due to different measurements cannot significantly change the intrinsic relationship among the parameters listed in Table I. As shown in Fig. 1, there seems no clear linear relation between fragility and $K$ [or $G, K / G$, and the Poisson ratio, $\nu=1 / 2-3 /(6 K / G+2)$ ] just as Battrzzati ${ }^{7}$ and Johari ${ }^{9}$ observed. However, the scrupulous examination shows that a parameter combination, $m R T_{g} /(M / \rho)$, exhibits a striking linear correlation with $K$ (see Fig. 2) regardless of chemical components, structural details, etc., and the best fit of data is given by

$$
m R T_{g} /(M / \rho)-0.729=0.198 K,
$$

where $M / \rho$ is the molar volume $V_{m}$. That is, the correlation between $m$ and $K$ does not follow a simple linear relation, and the influence of material parameters such as $T_{g}, M$, and $\rho$ should be involved.

\section{THEORY}

Potential energy landscape (PEL) is an ideal method to describe the behavior of disordered systems, ${ }^{32,33}$ which can be used to reveal the underlying physics of the linear correlation in Eq. (2). For simplicity, let us assume that the potential energy of a system with $N$ pointlike constituent particles only depends on the spatial location $\mathbf{r}_{i}$ for each particle in a $(3 N+1)$-dimensional hypersurface. Intuitively, the state of 
TABLE I. Physical properties of 26 metallic glasses, such as density $\rho$, molar mass $M$, bulk modulus $K$, shear modulus $G$, glass transition temperature $T_{g}$, and fragility $m$.

\begin{tabular}{|c|c|c|c|c|c|c|c|c|}
\hline & Metallic glasses & $\begin{array}{c}\rho \\
\left(\mathrm{g} / \mathrm{cm}^{3}\right)\end{array}$ & $\begin{array}{c}M \\
(\mathrm{~g} / \mathrm{mol})\end{array}$ & $\begin{array}{c}K \\
(\mathrm{GPa})\end{array}$ & $\begin{array}{c}G \\
(\mathrm{GPa})\end{array}$ & $\begin{array}{c}T_{g} \\
(\mathrm{~K})\end{array}$ & $m$ & Refs. \\
\hline 1 & $\mathrm{Ce}_{70} \mathrm{Al}_{10} \mathrm{Ni}_{10} \mathrm{Cu}_{10}$ & 6.67 & 113 & 27 & 11.5 & 359 & 21 & 7,12 , and 13 \\
\hline 2 & $\mathrm{Cu}_{46} \mathrm{Zr}_{46} \mathrm{Al}_{8}$ & $7.23^{\mathrm{a}}$ & 73.3 & 116.4 & 34.3 & 701 & 43 & 15 \\
\hline 3 & $\mathrm{Cu}_{46} \mathrm{Zr}_{46} \mathrm{Al}_{7} \mathrm{Gd}_{1}$ & 7.40 & 74.6 & 123.7 & 32.9 & 720 & 29 & 15 \\
\hline 4 & $\mathrm{Fe}_{70} \mathrm{P}_{10} \mathrm{C}_{5} \mathrm{~B}_{5} \mathrm{Si}_{3} \mathrm{Al}_{5} \mathrm{Ga}_{2}$ & $6.24^{\mathrm{b}}$ & 46.9 & 113.4 & 58.5 & $736^{\mathrm{c}}$ & 34 & 15 \\
\hline 5 & $\mathrm{Ho}_{39} \mathrm{Al}_{25} \mathrm{Co}_{20} \mathrm{Y}_{16}$ & $6.50^{\mathrm{b}}$ & 97.1 & 63.60 & 26.2 & 630 & 49 & 15 \\
\hline 6 & $\mathrm{La}_{55} \mathrm{Al}_{25} \mathrm{Cu}_{10} \mathrm{Ni}_{5} \mathrm{Co}_{5}$ & 6.00 & 95.4 & 44.2 & 15.6 & 430 & 28 & 13,15 , and 19 \\
\hline 7 & $\mathrm{Nd}_{60} \mathrm{Fe}_{20} \mathrm{Co}_{10} \mathrm{Al}_{10}$ & 7.00 & 106 & 46.54 & 19.44 & 493 & 33 & 15,17 , and 19 \\
\hline 8 & $\mathrm{Pd}_{39.1} \mathrm{Ni}_{10.1} \mathrm{Cu}_{29.9} \mathrm{P}_{20.9}$ & 9.2 & 73.0 & 158.7 & 35.2 & 576 & 52 & 15 \\
\hline 9 & $\mathrm{Pd}_{39} \mathrm{Ni}_{10} \mathrm{Cu}_{30} \mathrm{P}_{21}$ & 9.152 & 72.9 & 159.1 & 35.1 & 586 & 55 & 15 and 17 \\
\hline 10 & $\mathrm{Pd}_{40} \mathrm{Ni}_{10} \mathrm{Cu}_{30} \mathrm{P}_{20}$ & 9.3 & 73.7 & 146 & 33 & 560 & 52 & 13,20 , and 21 \\
\hline 11 & $\mathrm{Pd}_{40} \mathrm{Ni}_{40} \mathrm{P}_{20}$ & 9.405 & 72.2 & 185 & 38.6 & 602 & 54 & 15,19, and 22 \\
\hline 12 & $\mathrm{Pd}_{48} \mathrm{Ni}_{32} \mathrm{P}_{20}$ & 9.83 & 76 & 176.7 & 36.2 & 590 & 48 & 15 and 17 \\
\hline 13 & $\mathrm{Pd}_{64} \mathrm{Ni}_{16} \mathrm{P}_{20}$ & 10.1 & 83.7 & 166 & 32.7 & 582 & 51 & 13,21 , and 23 \\
\hline 14 & $\mathrm{Pd}_{77.5} \mathrm{Cu}_{6} \mathrm{Si}_{16.5}$ & 10.4 & 90.9 & 167 & 31.5 & 635 & 52 & 7,23 , and 24 \\
\hline 15 & $\operatorname{Pr}_{60} \mathrm{Al}_{10} \mathrm{Ni}_{10} \mathrm{Cu}_{20}$ & 6.90 & 106 & 45.2 & 13.6 & 409 & 31 & 20 and 25 \\
\hline 16 & $\mathrm{Pt}_{60} \mathrm{Ni}_{15} \mathrm{P}_{25}$ & 15.7 & 133 & 202 & 33.8 & 500 & 86 & 13 and 26 \\
\hline 17 & $\mathrm{Mg}_{65} \mathrm{Cu}_{25} \mathrm{Y}_{10}$ & 3.978 & 40.6 & $44.71^{\mathrm{d}}$ & $19.6^{\mathrm{d}}$ & 402 & 41 & 7,21 , and 23 \\
\hline 18 & $\mathrm{Mg}_{65} \mathrm{Cu}_{25} \mathrm{Gd}_{10}$ & 4.04 & 47.4 & 46.3 & 18.6 & 417 & 41 & 13 and 15 \\
\hline 19 & $\mathrm{Mg}_{65} \mathrm{Cu}_{25} \mathrm{~Tb}_{10}$ & 3.98 & 47.4 & 44.7 & 19.6 & 414 & 47 & 15 and 17 \\
\hline 20 & $\mathrm{Ni}_{60} \mathrm{Nb}_{35} \mathrm{Sn}_{5}$ & 8.64 & 74.3 & 267 & 66.32 & 885 & 70 & 15 and 17 \\
\hline 21 & $\mathrm{Ni}_{64} \mathrm{Pd}_{16} \mathrm{P}_{20}$ & 8.75 & 61.3 & 169.8 & 37.9 & 587 & 50 & 15 and 17 \\
\hline 22 & $\mathrm{Zr}_{41} \mathrm{Ti}_{14} \mathrm{Cu}_{12.5} \mathrm{Ni}_{10} \mathrm{Be}_{22.5}$ & 6.12 & 59.9 & 114.7 & 37.4 & 627 & 50 & 7,17 , and 21 \\
\hline 23 & $\mathrm{Zr}_{41.2} \mathrm{Ti}_{13.8} \mathrm{Cu}_{12.5} \mathrm{Ni}_{10} \mathrm{Be}_{22.5}$ & 5.9 & 60.0 & 114.1 & 34.1 & 618 & 50 & 13 and 27 \\
\hline 24 & $\mathrm{Zr}_{46.75} \mathrm{Ti}_{8.25} \mathrm{Cu}_{7.5} \mathrm{Ni}_{10} \mathrm{Be}_{27.5}$ & 6.01 & 59.5 & 113.38 & 35.2 & 590 & 44 & 15,19 , and 27 \\
\hline 25 & $\mathrm{Zr}_{55} \mathrm{Al}_{19} \mathrm{CO}_{19} \mathrm{Cu}_{7}$ & 6.2 & 101.7 & 114.9 & 37.6 & 733 & $72^{\mathrm{e}}$ & 15 and 17 \\
\hline 26 & $\mathrm{Zr}_{65} \mathrm{Al}_{10} \mathrm{Ni}_{10} \mathrm{Cu}_{15}$ & $6.75^{\mathrm{f}}$ & 77.4 & 106.65 & 30.27 & 653 & 30 & 15,19 , and 28 \\
\hline
\end{tabular}

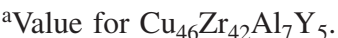

${ }^{\mathrm{b}}$ Calculated by atom mole ratio.

${ }^{c}$ Value for $\mathrm{Fe}_{80} \mathrm{P}_{13} \mathrm{C}_{7}$.

such a system can be represented by a point $s$ on or above the hypersurface. By analogy to Earth's topographic maps, Stillinger and Weber provided a formally exact portioning of the configurational space as a sum of distinct basins, associating with each local minimum of the potential energy surface, namely, an inherent structure (IS). The purpose is to assign any configuration of atoms uniquely to one local minimum by the steepest descent path; if it is not at a minimum, the displacement exhibited by the system is simply regarded as a "vibrational," possibly anharmonic in character, displacement. Thus, packing and vibration effects can be cleanly separated.

The system free energy in an IS can be expressed as a sum of a configurational contribution, accounting for the number of the available basins, and a vibrational one, expressing the free energy of the system when constrained in one of the basins. ${ }^{34,35}$ Here, the Helmholtz free energy $F(T, V)$ of a metallic glass (unit mass) at the temperature below $T_{g}$ can also be written as
${ }^{\mathrm{d}}$ Values for $\mathrm{Mg}_{65} \mathrm{Cu}_{25} \mathrm{~Tb}_{10}$.

eValue for $\mathrm{Zr}_{55} \mathrm{Al}_{22.5} \mathrm{Co}_{22.5}$.

${ }^{\text {fValue for }} \mathrm{Zr}_{65} \mathrm{Cu}_{17.5} \mathrm{Al}_{7.5} \mathrm{Ni}_{10}$.

$$
F(T, V)=F_{\text {conf }}(T, V)+F_{\text {vib }}(T, V),
$$

where the first term on the right hand side is the configurational portion denoted as $F_{\text {conf }}=U(V)-T S_{\text {conf }}$, with $U(V)$ the average specific potential energy of internal energy and $-T S_{\text {conf }}$ the configurational contribution to $F_{\text {conf }}$ that switches off due to $T<T_{g}$. The second term, $F_{v i b}=U_{v i b}-T S_{v i b}$, indicates the vibrational part, where $S_{v i b}$ is the vibrational entropy due to departure from the local minimum and the kinetic part of internal energy $U_{v i b}$ can be negligible. Since a metallic glass is an isotropic body, its potential energy can be assumed as ${ }^{36}$

$$
U(V)=-A / V^{\alpha / 3}+B / V^{\beta / 3} \quad(\alpha>\beta),
$$

where $A$ and $B$ are constants. Here, the first term represents the energy of the attractive interaction and the second term is the energy of repulsion. The values of $\alpha$ and $\beta$ are determined by material composition and structure. A glass at tem- 

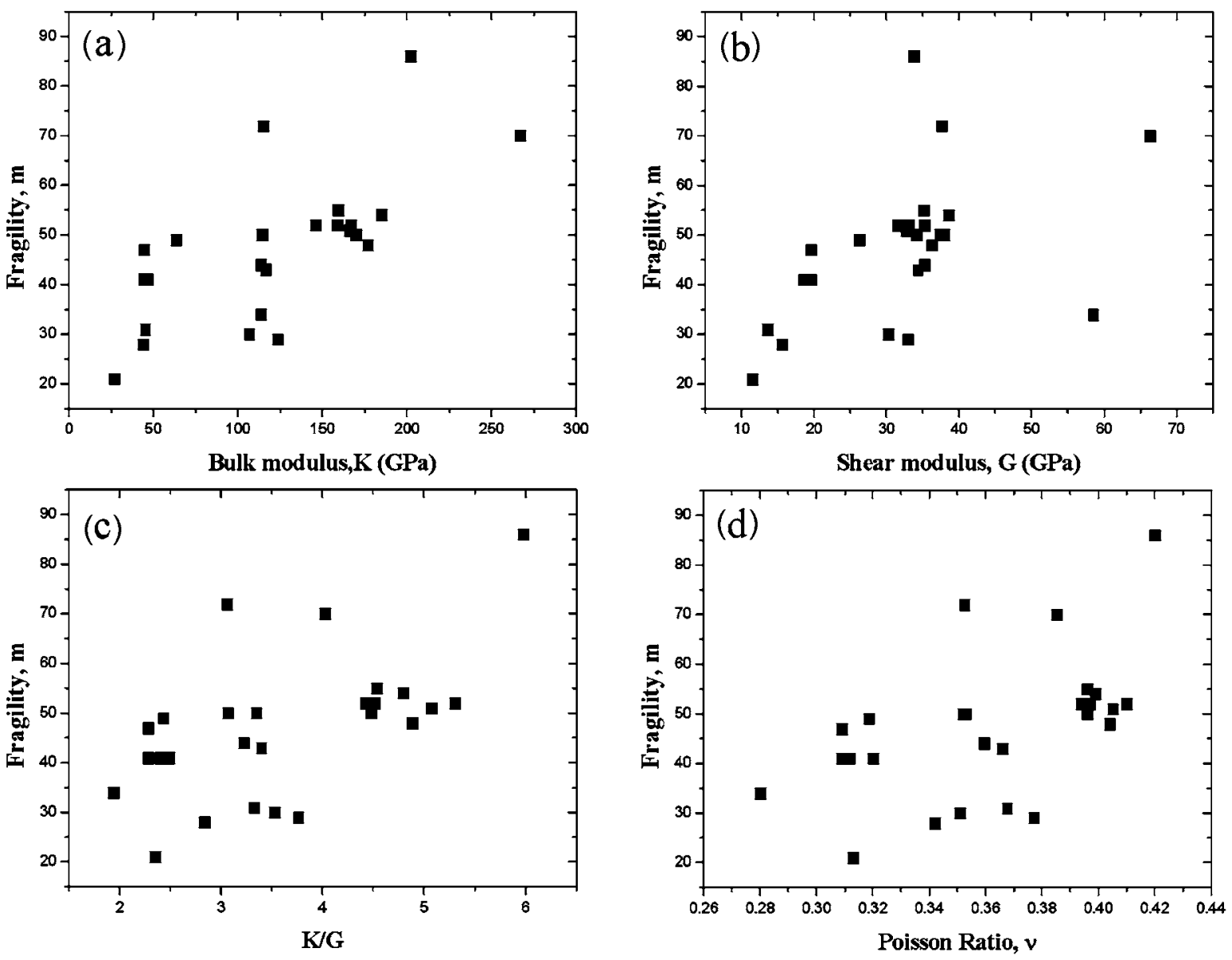

FIG. 1. Fragility versus (a) bulk modulus $K$, (b) shear modulus $G$, (c) bulk-shear modulus ratio, $K / G$, and (d) Poisson ratio $v$.

perature below $T_{g}$ is at or near a potential energy minimum or an IS. Its potential energy is $U_{0}=U\left(V_{0}\right)$ due to $(\partial U / \partial V)_{V_{0}}=0$. According to the thermodynamics, ${ }^{37}$ the isothermal bulk modulus at $T<T_{g}$ can be obtained by

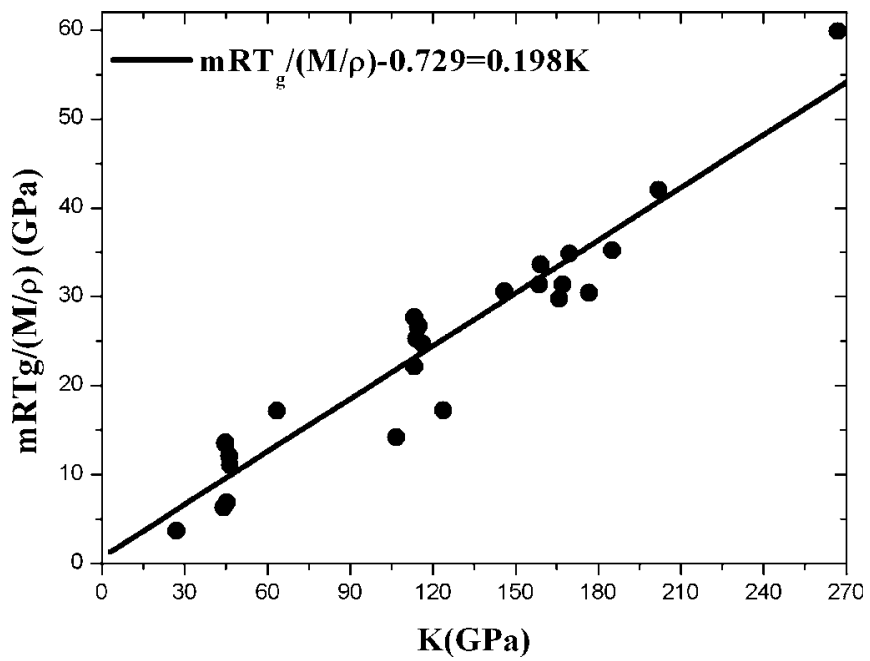

FIG. 2. Correlation between $m R T_{g} /(M / \rho)$ and bulk modulus $K$ in the glassy state, where the straight line is the best fitting results by Eq. (2).

$$
K=-\alpha \beta \rho U_{0} / 9-(T / \rho)\left(\partial^{2} S_{v i b} / \partial V^{2}\right)_{T, V_{0}} .
$$

Note that Eqs. (3) and (4) are used in deriving this equation. Many studies $^{38}$ have demonstrated that the viscositytemperature relation of supercooled liquids for metallic glass formers, especially approaching $T_{g}$, can be described well by the VFT equation. ${ }^{39}$ In terms of $U_{0}$, the VFT equation can be expressed as ${ }^{40,41}$

$$
\eta=\eta_{\infty} \exp \left\{-l M U_{0} /\left[R\left(T-T_{0}\right)\right]\right\},
$$

where $l$ is a numerical factor, $R$ is a gas constant, and $T_{0}$ is the Vogel temperature. Compared to the usual expression of the VFT equation, $\eta=\eta_{\infty} \exp \left[B /\left(T-T_{0}\right)\right]$, where $B$ is the activation energy of viscous flow, we find $B=-l M U_{0} / R$. So, Eq. (6) implies that the activation energy of viscous flow when $T>T_{0}$ is proportional to the IS potential energy $U_{0}$. Based on this assumption, Gemant ${ }^{41}$ established a relation between bulk modulus and viscosity of plastics. Applying the obtained relation to glass materials exhibits a good correlation between room-temperature elastic modulus and viscosity at high temperature. ${ }^{41}$ Also, the assumption is consistent with the basic tenet of PEL, namely, "the existence of potential energy barriers large compared to thermal energy are intrinsic to the occurrence of the glassy state, and dominate flow, at least at low temperature. ${ }^{32}$ Inserting Eq. (6) into Eq. 


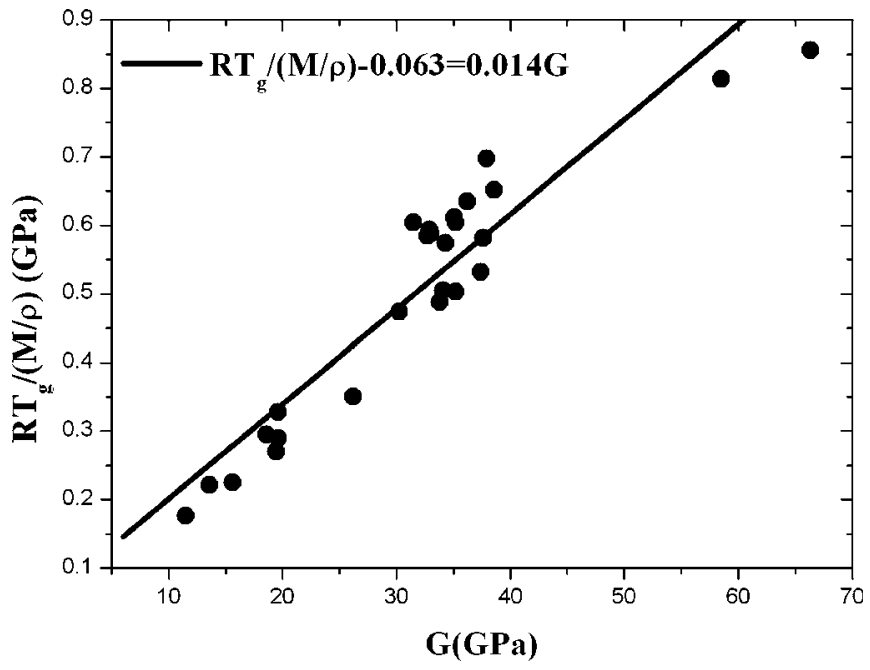

FIG. 3. Correlation between $R T_{g} /(M / \rho)$ and shear modulus $G$ in the glassy state, where the straight line is the best fitting results by Eq. (8).

(1) yields $U_{0}=-m R\left(T_{g}-T_{0}\right)^{2} \ln 10 /\left(l M T_{g}\right)$. Further, according to Eq. (5) and $T_{g}=T_{0}\left[1+B /\left(17 T_{0} \ln 10\right)\right],{ }^{1}$ we have the following correlation between fragility and bulk modulus:

$$
m R T_{g} /(M / \rho)+L(T)=C K,
$$

where $\quad L(T)=C\left[-(T / \rho)\left(\partial^{2} S_{v i b}\right) / \partial V^{2}\right]_{T, V_{0}} \quad$ and $\quad C=9 l[1$ $\left.+17\left(T_{0} / B\right) \ln 10\right] /(\alpha \beta \ln 10)$. Surprisingly, Eq. (7) is totally identical to the empirical fitting, i.e., Eq. (2), so long as $L(T)=-0.729 \mathrm{GPa}$ and $C=0.198$. It is important to point out that Eq. (7) is valid if temperature is below $T_{g}$, and Eq. (6) is only valid when $T>T_{0}$. The term $L(T)$ reflects the temperature softening effect on bulk modulus, and $C$ is weakly material dependent for metallic glasses according to the fit to experimental data.

\section{DISCUSSION}

\section{A. Fragility and bulk-shear modulus ratio}

To shed an insight into whether fragility $m$ is correlated linearly with $K / G$, we examined the data in Table I. A striking linear correlation between $R T_{g} /(M / \rho)$ and shear modulus $G$ is observed, as shown in Fig. 3. This linear correlation can be best fitted by

$$
R T_{g} /(M / \rho)+H(T)=D G,
$$

where $H(T)=-0.063 \mathrm{GPa}$, which is temperature dependent like the term $L(T)$ in Eq. (7), and $D=0.014$. It is worth noting that this intrinsic relation for metallic glasses is consistent with those obtained by Johnson and Somwer ${ }^{13}$ and Yang et $a l .{ }^{16}$ Now, we realize that although the correlation between fragility $m$ and bulk modulus $K$ is characterized explicitly by Eq. (7), the correlation between fragility $m$ and shear modulus $G$ is characterized implicitly by this equation through the parameter $R T_{g} /(M / \rho)$ that is scaled linearly with $G$, as shown in Fig. 3. Combining Eq. (7) with Eq. (8), we have the following correlation:

$$
m=a(K / G)+b(T ; m, G),
$$

where $a=C / D$ is a material-independent constant and $b(T ; m, G)=[m H(T)-L(T)] /(D G)$ indicates the effect of temperature on modulus. It is easy to see that the linear correlation between $m$ and $K / G$ in Eq. (9) exists only when $b(T ; m, G)$ is a material-independent constant. However, this condition is too harsh given that both $m$ and $G$ are highly material dependent. Obviously, $m$ must be strictly proportional to $K / G$ for solid glasses at absolute zero temperature or very high frequency. Each of these two conditions leads to $L(T)=0$ and $H(T)=0$. In fact, the former condition, i.e., the observed correlation at absolute zero temperature, is consistent with the finding of Scopigno $\mathrm{et} \mathrm{al.}^{3}$ (vide post), while the latter implies that fragility is linearly linked to the instantaneous bulk-shear modulus ratio. ${ }^{5}$ Both the conditions require that measurements of the elastic constants of glasses be made either at a temperature low enough that molecular motion in structure is kinetically frozen or at a frequency used for ultrasonic measurements high enough that the temperaturedependent fast $\beta$ relaxational contributions (not only the structure relaxation ${ }^{5}$ ) are negligible. However, it is almost impossible that the above conditions are strictly satisfied, because, in a real experiment, sound velocity measurements on modulus in the frequency range of $20-50 \mathrm{~Hz}$ may not yield elastic properties attributable to vibrations alone, while measurements on solid glasses are usually performed at room temperature. This is why the experimental data do not obey the linear correlation. ${ }^{7,9}$ Our finding that fragility is linearly related to the zero temperature or much higher frequency bulk-shear modulus ratio implies that liquid viscosity is determined by its zero temperature or short-time-scale elastic properties. This seems to be at odds with the prevailing paradigm of glass science, ${ }^{10}$ which is embodied in the Adam-Gibbs model. ${ }^{42}$ Nevertheless, according to elastic models of glass forming, the barrier transition for a "flow event" (an atomic rearrangement) does take place on a very short time scale. ${ }^{43}$ Furthermore, both Egami's atomistic theory ${ }^{8}$ and the microstructural model of Ichitsubo et al. ${ }^{6}$ can perfectly explain the Novikov-Sokolov observations. Actually, in their models, the temperature-dependent modulus was neglected, which corresponds to $b(T ; m, G) \equiv 0$ in our case.

\section{B. Shear-induced dilatation}

Next, let us discuss the physical pictures behind Eqs. (7) and (8). In soil mechanics, it has been known for years that shear of randomly close-packed grains causes dilatation. The same phenomenon can be applied at the atomic scale. Crystals can deform at constant volume because the periodicity along slip planes provides identical atomic positions for sheared materials. A sheared portion of an amorphous material, on the other hand, does not find such a perfect fit and thus will leave some holes. ${ }^{44}$ It has been recognized that macroscopic flow of an amorphous material occurs as a result of a number of individual atomic jumps or flow events. Thus, a real flow event in bulk metallic glasses must result in shear deformation and an accompanying bulk deformation 
(or dilatation). Actually, according to Dyre's shoving model, spherical symmetry in real flow events is probably violated, leading to some compression of surroundings and a contribution to activation energy that is proportional to bulk modulus. ${ }^{45}$ The equivalent PEL of the shoving model also confirmed this point; thereby, activation energy must involve both shear and bulk moduli. ${ }^{46}$ However, which of them controls the activation energy? The elastic models such as rate theory, shoving model, etc., give a theoretical answer and clarify that activation energy is proportional to shear modulus that is a controlling parameter in a real flow event. ${ }^{43} \mathrm{It}$ is of interest to note that our results are consistent with the elastic models from the viewpoint of experimental data for bulk metallic glasses (BMGs). Equation (8) characterizes shear deformation in real flow events, while Eq. (7) determines shear-induced bulk deformation. Based on the physical analogy between the shear transformation zone deformation and glass transition, Yang et al. found that the shear yield strength of BMGs at ambient temperature $T_{0}$ can be predicted well by a unified parameter, $R\left(T_{g}-T_{0}\right) / V_{m} .{ }^{16} \mathrm{Com}-$ pared to Eq. (8), we find that a compound parameter, $R T_{g} / V_{m}$, can be regarded as a scale on shear yield strength at zero temperature, although $H(T)$ is weakly dependent on $T_{0}$. Data fitting shows that $D$ is a material-independent constant with magnitude of $10^{-2}$, which is consistent with the average shear yield strain $\gamma_{C}$ observed by Johnson and Samwer. ${ }^{13}$ Thus, $D$ can be considered as an apparent shear yield strain $\hat{\gamma}_{C}$. The linear correlation between $R T_{g} / V_{m}$ and $G$ implies that $G$ is a controlling quantity in real flow events. In parallel with this consideration, it is natural to investigate the relationship between $R T_{g} / V_{m}$ and $K$. Although $m R T_{g} / V_{m}$ is proportional to $K, R T_{g} / V_{m}$ does not scale with $K$, i.e., $\left(R T_{g} / V_{m}\right) / K \sim \hat{\xi}_{c} \neq$ const, where $\hat{\xi}_{C}=C / m$ is shear-induced dilatation (with the order of magnitude of $10^{-3}$ ) while shear yielding occurs. It is noted that $\hat{\xi}_{C}$ is associated with $m$, and thus highly material specific. Since $C$ is a materialindependent constant for metallic glasses, it is easily seen that fragility $m$ is in inverse proportional to $\hat{\xi}_{C}$. This means that, subjected to same hydrostatic compression, strong glasses are of higher bulk dilatation, while fragile ones have smaller bulk deformation. ${ }^{47}$ The explicit correlation between bulk (not shear) modulus and fragility may result from this highly material-dependent bulk deformation in BMGs. ${ }^{17}$

Using a model developed by Knuyt et al. based on a Gaussian distribution for the nearest-neighbor distance in an ideal unicomponent metallic glass, ${ }^{48}$ we can approximately calculate the ratio of bulk to shear elastic energy in a flow event with the following equation:

$$
\frac{E_{\text {elastic,bulk }}}{E_{\text {elastic,shear }}}=\frac{(1 / 2) \hat{\xi}^{2} K}{(3 / 2) \hat{\gamma}^{2} G} \text {. }
$$

Note that this ratio increases with decreasing $m$ and increasing $K / G$. However, there is a tendency for fragility $m$ to increase with increasing $K / G$, and vice versa. For BMGs (see Table I), $m$ mostly ranges from 30 to 80 , and $K / G$ is in the range 2-5; thus, the range of this ratio of bulk to shear elastic energy in a flow event is usually less than about $10 \%$.
Our result is consistent with the result of $8 \%$ calculated by Dyre and Olsen based on PEL $^{46}$ or the $10 \%$ calculated recently by Dyre on a standard dipole expansion. ${ }^{49}$ It indicates that the bulk modulus' contribution to the activation energy is much less than that due to shear deformation in a real flow event. Consequently, shear (not bulk) modulus controls the activation energy (that is shear dominant) in a real flow event.

\section{Comparison with related works}

At this point, we briefly discuss related works. Based on local topological instability analysis and the assumption that bulk modulus is almost independent of temperature, Egami ${ }^{14}$ predicts that there is a correlation between $T_{g}$ and bulk modulus $K$ for metallic glasses, $T_{g}=6.14 \times 10^{-3} K\langle\Omega\rangle / k_{B}$. Actually, this assumption is equivalent to the case that the temperature-dependent term $L(T) \rightarrow 0$ in Eq. (7). Thus, Eq. (7) can be converted into

$$
T_{g}=(C / m)\left(K\langle\Omega\rangle / k_{B}\right),
$$

where $\langle\Omega\rangle=M /\left(N_{0} \rho\right)$ is the average atomic volume of a metallic glass with $N_{0}$ the Avogadro constant. It is interesting to find that if the fragility value in Eq. (11) is equal to 32.25 (the fragility value of metallic glasses ranges from 21 to 86 in this study), Egami's prediction is recovered.

Also, Eq. (7) leads us directly to an explanation of the striking finding of Scopigno et al. ${ }^{3}$ They found that fragility is proportional to a dimensionless quantity $\alpha$, i.e., the temperature steepness of the nonergodicity factor at $T \rightarrow 0 .{ }^{12}$ The low-temperature $\alpha$ only depends on the interaction potential and disordered structure. In the harmonic approximation for vibrational dynamics, $\alpha$ can be expressed as ${ }^{3} \quad \alpha$ $=\left(M N \Sigma_{p} \omega_{p}^{-2}\right) /\left(k_{B} T_{g} Q^{2}\right)$, where $M$ is the molecular mass, $k_{B}$ is the Boltzmann constant, $N$ is the number of particles, $Q$ is the wave vector, and $p$ is summed over $3 N$ normal modes. In a system consisting of $N_{0}$ (the Avogadro constant), $\alpha$ can be converted into $\alpha=(M / \rho)\left\{\rho /\left[Q^{2}\left(1 / N_{0}\right) \Sigma_{p} \omega_{p}^{-2}\right]\right\} /\left(R T_{g}\right)$, where $Q^{2}\left(1 / N_{0}\right) \Sigma_{p} \omega_{p}^{-2} \propto v_{l}^{-2}$ with $v_{l}$ the longitudinal sound velocity. ${ }^{50}$ Because $K \propto \rho v_{l}^{2}$, where $v_{l}$ is proportional to the transverse sound velocity $v_{t},{ }^{3} \alpha$ reduces to

$$
\alpha \propto(M / \rho) K /\left.\left(R T_{g}\right)\right|_{T \rightarrow 0} .
$$

At $T \rightarrow 0$, Eq. (8) becomes $R T_{g} /(M / \rho) \propto G$. Substituting it into Eq. (12) gives $\alpha \propto(K / G)_{T=0}$. Due to $m \propto(K / G)_{T=0}$, as discussed above, the finding of Scopigno et al., i.e., $m \propto \alpha$, is also recovered. It is easy to see that the low-temperature $\alpha$ can also be determined by elastic modulus at zero temperature. However, modulus measurements on metallic glasses are usually performed at room temperature. So, it is impossible to calculate the low-temperature $\alpha$ by using the data listed in Table I, whereas Scopigno et al. ${ }^{3}$ cleverly derived the parameter $\alpha$ from the IXS data available for glasses at low temperature.

Finally, to survey the universality of two correlations in Eqs. (7) and (8), let us consider 11 nonmetallic glasses, including $\mathrm{BeF}_{2}, \mathrm{~B}_{2} \mathrm{O}_{3}, \mathrm{SiO}_{2}, \mathrm{GeO}_{2}, \mathrm{AS}_{2} \mathrm{~S}_{3}, \mathrm{CKN}$, glycerol, salol, $m$-toluidine, OTP, and $m$-TCP. ${ }^{3,5}$ As shown in Fig. 4, 


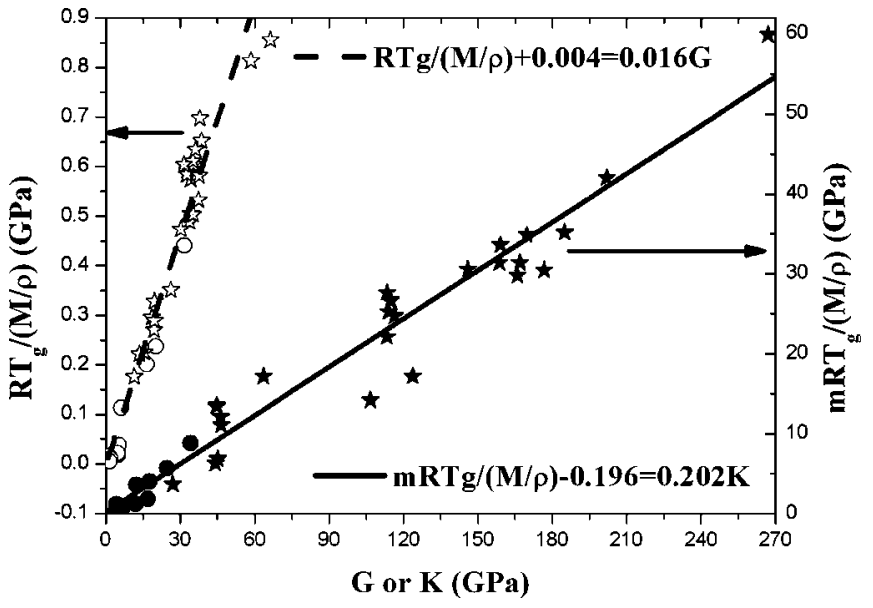

FIG. 4. $m R T_{g} /(M / \rho)$ versus $K$ and $R T_{g} /(M / \rho)$ versus $G$ for metallic and nonmetallic glasses. Black and open stars: metallic glasses; black and open circles: nonmetallic glasses.

the linear relationship indicates that the observed intrinsic correlations might be more general although the fitting parameters are not constants. Unfortunately, there are not enough glass formers whose relevant data are all known.
Thus, further work is needed to check the universality of these intrinsic correlations.

\section{CONCLUSIONS}

In conclusion, an intrinsic correlation between fragility and bulk modulus for 26 metallic glasses was revealed, which could be rationalized in terms of PEL thermodynamics. We found that fragility (the degree of non-Arrhenius viscosity of liquids) can be determined by zero-temperature or short-time-scale mechanical properties of solid glasses. Moreover, an inverse linear correlation between fragility and bulk dilatation induced by shear-dominant activation energy was discovered. These observed intrinsic correlations may be extended to nonmetallic glasses. We believe that these findings are of importance for understanding relationships between microstructure and mechanical properties in BMGs.

\section{ACKNOWLEDGMENTS}

L.H. is indebted to F. Spaepen for valuable discussions. This work was supported by the National Science Foundation of China (Nos. 10472119 and 10232040), the key project of the Chinese Academy of Sciences (Nos. KJCXSW-L08 and KJCX2-YW-M04), and the K. C. Wong Education Foundation, Hong Kong.
*Corresponding author; lhdai@lnm.imech.ac.cn

${ }^{1}$ C. A. Angell, J. Non-Cryst. Solids 73, 1 (1985); 131-133, 13 (1991); R. Böhmer, K. L. Ngai, C. A. Angell, and D. J. Plazek, J. Chem. Phys. 99, 4201 (1993); C. A. Angell, Science 267, 1924 (1995).

${ }^{2}$ A. P. Sokolov, E. Rössler, A. Kisliuk, and D. Quitmann, Phys. Rev. Lett. 71, 2062 (1993); K. Ngai and O. Yamamuro, J. Chem. Phys. 23, 10403 (1999); L.-M. Martinez and C. A. Angell, Nature (London) 410, 663 (2001); S. Venkataraman, K. Biswas, B. C. Wei, D. J. Sordelet, and J. Eckert, J. Phys. D 39, 2600 (2006).

${ }^{3}$ T. Scopigno, G. Ruocco, F. Sette, and G. Monaco, Science 302, 849 (2003); U. Buchenau and A. Wischnewski, Phys. Rev. B 70, 092201 (2004).

${ }^{4}$ Yong Li, H. Y. Bai, W. H. Wang, and K. Samwer, Phys. Rev. B 74, 052201 (2006).

${ }^{5}$ V. N. Novikov and A. P. Sokolov, Nature (London) 431, 961 (2004).

${ }^{6}$ T. Ichitsubo, E. Matsubara, T. Yamamoto, H. S. Chen, N. Nishiyama, J. Saida, and K. Anazawa, Phys. Rev. Lett. 95, 245501 (2005).

${ }^{7}$ L. Battrzzati, Mater. Trans. 46, 2915 (2005).

${ }^{8}$ T. Egami, Intermetallics 14, 882 (2006).

${ }^{9}$ G. P. Johari, Philos. Mag. 86, 1567 (2006); Spyros N. Yannopoulos and G. P. Johari, Nature (London) 442, E7 (2006).

${ }^{10}$ J. C. Dyre, Nat. Mater. 3, 749 (2004).

${ }^{11}$ A. L. Greer, Science 267, 1947 (1995); M. F. Ashby and A. L. Greer, Scr. Mater. 54, 321 (2005).

${ }^{12}$ J. J. Lewandowski, W. H. Wang, and A. L. Greer, Philos. Mag. Lett. 85, 77 (2005).
${ }^{13}$ W. L. Johnson and K. Samwer, Phys. Rev. Lett. 95, 195501 (2005).

${ }^{14}$ T. Egami, Rep. Prog. Phys. 47, 1601 (1984); Mater. Sci. Eng., A 226-228, 261 (1997); S. P. Chen, T. Egami, and V. Vitek, Phys. Rev. B 37, 2440 (1988).

${ }^{15}$ W. H. Wang, J. Non-Cryst. Solids 351, 1481 (2005); J. Appl. Phys. 99, 1 (2006).

${ }^{16}$ B. Yang, C. T. Liu, and T. G. Nieh, Appl. Phys. Lett. 88, 221911 (2006).

${ }^{17}$ V. N. Novikov and A. P. Sokolov, Phys. Rev. B 74, 064203 (2006).

${ }^{18}$ At present, only 26 metallic glasses, whose relevant data are all known, can be found from the literature.

${ }^{19}$ Y. Kawamura, T. Nakamura, H. Kato, H. Mano, and A. Inoue, Mater. Sci. Eng., A 304-306, 674 (2001).

${ }^{20}$ Z. F. Zhao, Z. Zhang, P. Wen, M. X. Pan, D. Q. Zhao, W. H. Wang, and W. L. Wang, Appl. Phys. Lett. 82, 4699 (1983).

${ }^{21}$ L. N. Hu, X. F. Bian, W. M. Wang, G. R. Liu, and Y. B. Jia, J. Phys. Chem. B 109, 13737 (2005).

${ }^{22}$ E. F. Lambson, W. A. Lambson, J. E. Macdonald, M. R. J. Gibbs, G. A. Saunders, and D. Turnbull, Phys. Rev. B 33, 2380 (1986).

${ }^{23}$ D. N. Perera, J. Phys.: Condens. Matter 11, 3807 (1999).

${ }^{24}$ H. S. Chen, Scr. Metall. 9, 411 (1975).

${ }^{25} \mathrm{~S}$. Li et al., Sci. Technol. Adv. Mater. 6, 823 (2005).

${ }^{26}$ T. Komatsu, J. Non-Cryst. Solids 185, 199 (1995).

${ }^{27}$ Y. Zhao, X. F. Bian, K. B. Yin, J. K. Zhou, J. Y. Zhang, and X. X. Hou, Physica B 349, 327 (2004).

${ }^{28}$ Y. X. Wei, B. Zhang, R. J. Wang, M. X. Pan, D. Q. Zhao, and W. H. Wang, Scr. Mater. 54, 599 (2006).

${ }^{29}$ R. Busch, E. Bakke, and W. L. Johnson, Acta Mater. 46, 475 
(1998).

${ }^{30}$ K. Ito, C. T. Moynihan, and C. A. Angell, Nature (London) 398, 492 (1999).

${ }^{31}$ B. Zhang, R. J. Wang, D. Q. Zhao, M. X. Pan, and W. H. Wang, Phys. Rev. B 70, 224208 (2004).

${ }^{32}$ M. Goldstein, J. Chem. Phys. 51, 3728 (1969); F. H. Stillinger and T. A. Weber, Science 255, 983 (1984); F. H. Stillinger, ibid. 267, 1935 (1995); F. Sciortino, W. Kob, and P. Tartaglia, Phys. Rev. Lett. 83, 3214 (1999); P. G. Debenedetti and F. H. Stillinger, Nature (London) 410, 259 (2001).

${ }^{33}$ C. A. Angell, Nature (London) 393, 521 (1998).

${ }^{34}$ S. Sastry, Nature (London) 409, 164 (2001).

${ }^{35}$ E. LaNave, S. Mossa, and F. Sciortino, Phys. Rev. Lett. 88, 225701 (2002).

${ }^{36}$ R. H. Fowler, Statistical Mechanics (Macmillan, New York, 1936).

${ }^{37}$ Callen and B. Herbert, Thermodynamics (Wiley, New York, 1960).

${ }^{38}$ C. A. Volkert and F. Spaepen, Mater. Sci. Eng. 97, 449 (1988); W. L. Johnson, Mater. Res. Bull. 24, 42 (1999).

${ }^{39}$ H. Vogel, Phys. Z. 22, 645 (1921); G. S. Fulcher, J. Am. Ceram.
Soc. 8, 339 (1925); G. Tamman and W. Hesse, Z. Anorg. Allg. Chem. 156, 245 (1926).

${ }^{40}$ J. M. Burgers, Second Report on Viscosity and Plasticity (Nordemann, New York, 1938), Chap. I.

${ }^{41}$ A. Gemant, J. Appl. Phys. 12, 680 (1941).

${ }^{42}$ G. Adam and J. H. Gibbs, J. Chem. Phys. 43, 139 (1965).

${ }^{43}$ J. C. Dyre, Rev. Mod. Phys. 78, 953 (2006).

${ }^{44}$ F. Spaepen, Nat. Mater. 5, 7 (2006); J. Li, F. Spaepen, and T. C. Hufnagel, Philos. Mag. A 82, 2623 (2002).

45 J. C. Dyre, J. Non-Cryst. Solids 235-237, 142 (1998); J. C. Dyre, N. B. Olsen, and T. Christensen, Phys. Rev. B 53, 2171 (1996).

${ }^{46}$ J. C. Dyre and N. B. Olsen, Phys. Rev. E 69, 042501 (2004).

${ }^{47}$ W. H. Wang, P. Wen, L. M. Wang, Y. Zhang, M. X. Pan, D. Q. Zhao, and R. J. Wang, Appl. Phys. Lett. 79, 3947 (2001).

${ }^{48}$ G. Knuyt, L. De Schepper, and L. M. Stals, Philos. Mag. B 61, 965 (1990); G. Knuyt, L. M. Stals, and L. De Schepper, ibid. 63, 1289 (1991); G. Knuyt, and L. M. Stals, ibid. 64, 299 (1991).

${ }^{49}$ J. C. Dyre, Phys. Rev. B 75, 092102 (2007).

${ }^{50}$ G. Ruocco, F. Sette, R. Di Leonardo, G. Monaco, M. Sampoli, T. Scopigno, and G. Viliani, Phys. Rev. Lett. 84, 5788 (2000). 